(Aus der San Francisco Polyclinic, San Francisco, Calif., U. S. A.)

\title{
Ueber die Hervorrufung und Hemmung von Glykosurie in Kaninchen durch Salze.
}

\author{
Vierte Mittheilung: \\ Von
}

Dr. med. Martin H. Fischer.

(Mit 2 Textfiguren.)

\section{Einleitung.}

Es ist der Zweck der folgenden Seiten, eine ausführlichere Schilderung der Experimente über das obengenannte Thema, die ich in meiner dritten Mittheilung ${ }^{1}$ ) neulich in diesem Archiv nur in Auszug veröffentlicht habe, zu geben. Um die Versuche etwas verständlicher zu machen, will ich diese Schilderung mit einem kurzen Bericht über die früher von mir gefundenen hierher gehörigen Thatsachen einleiten, namentlich da meine ersten zwei Mittheilungen ${ }^{2}$ ) in einer etwas unzugänglichen Zeitschrift publicirt wurden.

Im Jahre 1871 beschrieben $\mathrm{Bock}$ und $\mathrm{Hoffmann}^{3}$ ) als eine neue Entstehungsart der Glykosurie die Einspritzung von grossen Quantitäten (25-30 ccm oder mehr pro fünf Minuten) einer $1 \%$ igen Kochsalzlösung in das Gefässsystem von Kaninchen. Ist der Versuch vorsichtig angestellt, so entsteht zunächst eine gesteigerte Harnsecretion, und einige Zeit später kommt im Harn Zucker zum Vorschein. Auch $\mathrm{Kü} \mathrm{Iz}^{4}$ ) scheint es geglückt zu sein, Glykosurie hierdurch

1) Martin H. Fischer, Pflüger's Archiv Bd. 106 S. 80.1904.

2) Martin H. Fischer, University of California Publications. Physiology vol. 1 p. 77. $190 \%$, und vol. 2 p. 87. 1904.

3) Bock und Hoffmann, Reichert und du Bois-Reymond's Archiv 1871 S. 550.

4) Külz, Eckhard's Beiträge Bd. 4 S. 177. 1872. Citirt nach Pflüger, Pflüger's Archiv Bd. 96 S. 313.1903.

E. Pflüger, Archiv für Physiologie. Bd. 109. 
hervorzurufen, ferner auch durch Einspritzung von Lösungen von essigsaurem Natrium, kohlensaurem Natrium, valeriansaurem Natrium und bernsteinsaurem Natrium in das Blut.

Die Einflössung von grossen Quantitäten dieser Salzlösungen in das Gefässsystem erzengt zwei Änderungen, - eine Zunahme der Wassermenge und eine Zunahme des Betrages an Natriumsalzen, die in dem Thiere enthalten sind. Es ist nun durch die Untersuchungen Loeb's gezeigt worden, dass durch eine Steigerung in der Concentration der Natriumionen die Reizbarkeit von Nerven und Muskeln erhöht wird, und dass rhythmische Zuckungen hervorgerufen werden. Weiter hat Lo eb zeigen können, dass Calciumsalze antagonistisch wirken, und dass durch Zusatz derselben die erregende Wirkung der Natriumsalze aufgehoben wird. Dr. Lo eb machte mich darauf aufmerksam, dass die Glykosurie in Kaninchen nach der Einflössung von Natriumsalzen möglicherweise eine Analogieerscheinung zur Hervorrufung von Muskelzuckungen durch dieselben Salze war, und dieser Vorschlag, für welchen ich Dr. Loeb auch an dieser Stelle wieder meinen besten Dank ausspreche, war der Ausgangspunkt der folgenden Versuche.

\section{Versuchsmethoden.}

Allgemeine Bemerkungen. Kräftige, gesunde Kaninchen wurden in allen folgenden Versuchen gebraucht. Die Thiere erbielten als Futter Gerste und Alfalfaheu sowie Mohrrüben. Die zuletzt genannte Nahrung ist aber für den Erfolg der Versuche nicht nothwendig.

Um die grossen Mengen Kochsalzlösung, die für die Hervorbringung der Glykosurie erforderlich sind, einzuspritzen, führten Bock und Hoffmann Canilen in das peripherische Ende der A. femoralis oder der A. carotis ein. Da dieses Verfahren eine bedeutende Verletzung des Thieres mit sich bringt und dem Experimentirenden nicht erlaubt, schnell von einem Blutgefäss zu einem anderen überzugehen, wenn die Canüle durch geronnenes Blut verstopft wird, ersetzte ich die Canüle durch zwei hypodermische Nadeln, die mit dem in Figur 1 gezeichneten Einspritzungsapparat in Verbindung standen.

$F$ ist ein Hahn der Wasserleitung der mittelst eines Gummischlauches mit der grossen Druckflasche $A$ verbunden ist. Wenn der Hahn geöffnet wird, wird die Luft in $A$ comprimirt und rückt durch 
die Gummischläuche $m$ und $m_{1}$ in die graduirten Cylinder $B$ und $C$. Die Cylinder $B$ und $C$ sind mit den Lösungen gefüllt, die in das Thier eingespritzt werden sollen. Der Druck der Luft aus $A$ auf den Spiegel dieser Lösungen veranlasst dieselben bei $N$ und $N_{1}$ auszufliessen, nachdem sie durch die Röhren $g n_{0}$ und $g_{1} n_{1} o_{1}$ gegangen sind. $N$ und $N_{1}$ stellen zwei hypodermische Nadeln dar, die an den

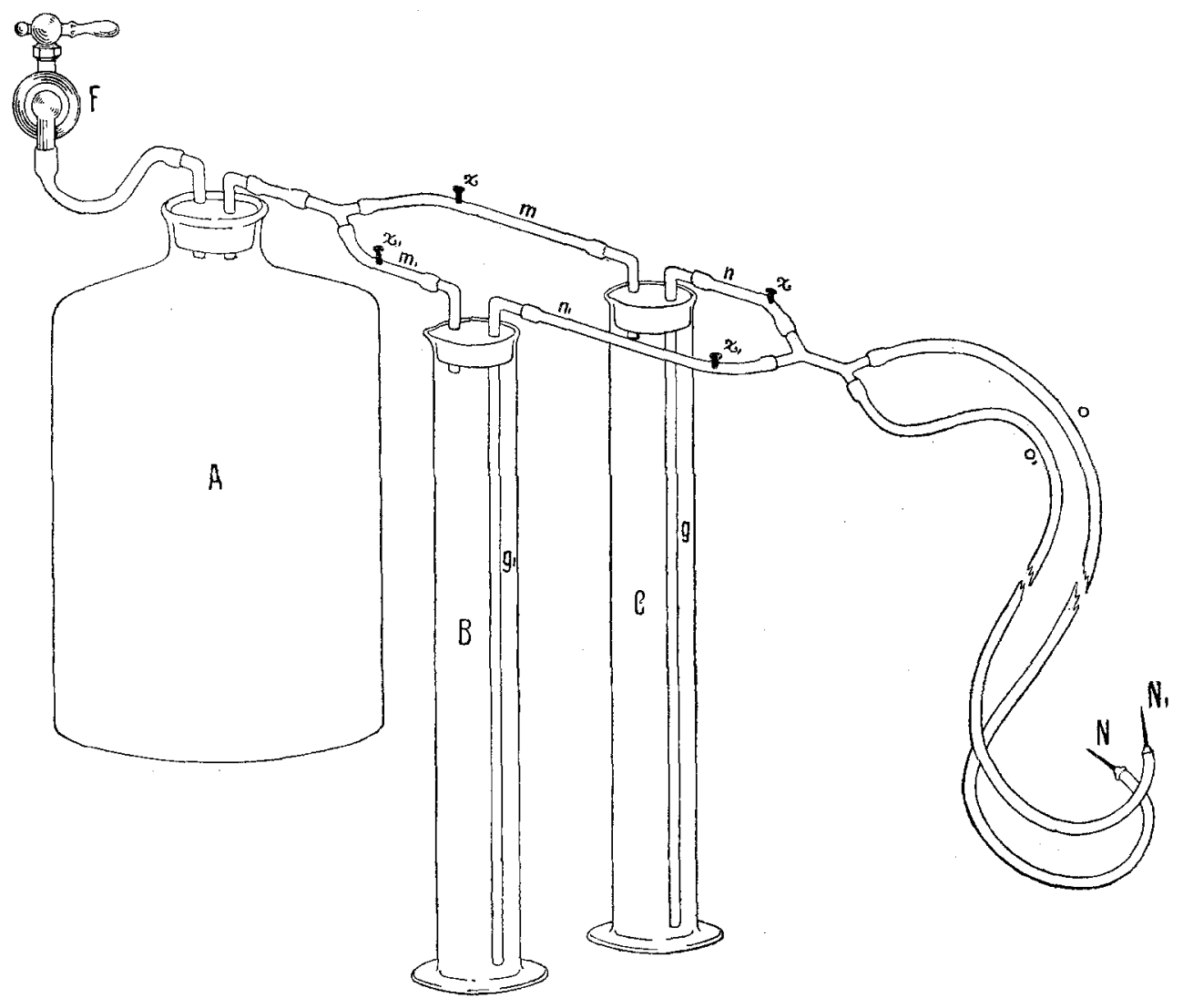

Fig. 1.

Gummischläuchen $o$ und $o_{1}$ befestigt sind. $x x$ und $x_{1} x_{1}$ bezeichnen die Schrauben von vier Schraubenquetschhähnen. Durch Schliessung von $x_{1}$ und $x_{1}$ bei Offenstehen von $x$ und $x$ kann nur die in $C$ enthaltene Lösung in die Röhren $o$ und $o_{1}$ übergehen. Durch passende Behandlung dieser Quetschhähne kann man folglich willkürlich von der Einspritzung der einen Lösung zu der Einspritzung einer anderen schnell übergehen, ohne im Uebrigen den Apparat oder das Thier zu stören. 
Die Einspritzungen der Salzlösungen wurden in die Seitenvenen der Ohren gemacht. Wenn durch Oedem des Ohres oder durch irgend eine andere Ursache es nöthig war, die Nadeln in einen anderen Theil des Körpers zu führen, so wurden sie in einen der oberflächlichen Zweige der V. femoralis, die an der inneren Seite der Lende liegen, und die bereits durch einen kleinen Hautschnitt blossgelegt wurden, eingesetzt. Diese Versuchsanordnung lässt die Thiere practisch unverletzt, ja ich habe mehrmals dasselbe Kaninchen an einem folgenden Tage wieder gebraucht. Unter diesen Umständen kann man wohl die Fehlerquellen als auf ein Minimum herabgesetzt ansehen. Die Kaninchen wurden auf dem Rücken liegend leicht befestigt und, um sie gegen Wärmeverlust zu schïtzen, in Watte und Handtücher eingewickelt. Die Salzlösungen wurden stets bis zur Körpertemperatur gewärmt, bevor sie eingespritzt wurden, und um sie warm zu halten, wurden die Cylinder und die Zuleitungsgummischläuche in warmes Wasser gelegt. In keinem der Experimente wurden Anesthetica benutzt, da diese an und für sich Glykosurie hervorrufen können ${ }^{1}$ ).

Der Urin wurde der Blase mittelst eines Katheters entnommen. Dies ist immer möglich bei Männchen. Bei den wenigen Weibchen, bei welchen dies nicht möglich war, wurde die Blase durch leichten Druck auf die Bauchwand entleert.

Zuckerbestimmung. Schon die Beobachtungen von Bock und Hoffmann und von $\mathrm{Kül} \mathrm{z}$ haben in eindeutiger Weise bewiesen, dass die reducirende Substanz, die im Harn nach Einflössung von reinen Natriumsalzlösungen zum Vorschein kommt, nichts anderes ist als Zucker, dass es sich wirklich um Traubenzucker im Harn handelt, habe ich dureh die Gärungs- und Phenylhydrazinprobe beweisen können. Zur quantitativen Bestimmung des Zuckers in meinen Experimenten gebrauchte ich eine normale Fehling'sche Lösung, die stets friseh gemischt und probirt wurde. Die Harnanalysen wurden jede $7^{1 / 2}$ Minuten gemacht, wenn eine genügende Menge Harn sich in dieser Zeit angesammelt hatte. Die

1) Auf die Versuche von O. H. Brown (Americ. Journ. of Physiol. vol. 10 p. 378. 1904) über Glykosurie durch Salzlösungen, die er seit dem Erscheinen meiner ersten zwei Mittheilungen über dasselbe Thema veröffentlicht hat, will ich hier nicht wieder eingehen. Für eine kurze Besprechung der Fehlerquellen in Brown's Experimenten siehe meine dritte Mittheilung. Pflüger's Archiv Bd. 106 S. 80.1904. 
Analysenmethode war folgende: Um das Eiweiss, das von Zeit zu Zeit zum Vorschein kommt, zu beseitigen, wurde jede Urinprobe durch Zusatz eines Tropfens Essigsäure schwach sauer gemacht und gekocht. Nach vorsichtiger Neutralisation mit Kaliumbydroxyd und dem Zusatz eines Ueberschusses bis zur schwach alkalischen Reaction wurde der Harn filtrirt. Wenn man nun abgemessene Mengen von Harn und abgemessene Mengen von Fehling"scher Lösung gebraucht, so kann man leicht eine Zunahme oder Abnahme in der Zuckerausscheidung wahrnehmen. In meinen ersten Versuchen genügte es mir zu wissen, ob die Zuckerausscheidung am Steigen oder Fallen war. In meinen späteren Experimenten versuchte ich, die absoluten Mengen des ausgeschiedenen Zuckers zu berechnen. Öfters jedoch musste ich mich wegen Mangel an Zeit damit befriedigen, festzustellen, ob die Menge des ausgeschiedenen Zuckers unter oder über einen gewissem Procentsatze lag.

\section{Ueber die Hervorrufung von Glykosurie durch Kochsalz- lösungen.}

Wenn 75-100 cem einer $1 / 6$ molecularen NaCl-Lösung pro 15 Minuten in die Blutbahn von kräftigen, gut ernährten Kaninchen eingespritzt werden, beobachtet man die folgenden Erscheinungen. In 15 Minuten nach dem Beginn der Einspritzung steigt die Menge des ausgeschiedenen Harns. Nach einer Stunde ist die Menge des Harns bis zu einem Punkt gestiegen, von dem an sie der Menge der eingespritzten Salzlösung ungefähr gleich läuft.

Die Eigenschaften des Harns werden ebenfalls stark geändert. Der Harn, der zu Anfang des Versuches trübe, tief gelb und gewöhnlich alkalisch auf Lackmuspapier reagirte, wird klar und hell im Laufe einer halben Stunde. Die Reaktion wird neutral oder Lackmuspapier gegenüber schwach sauer. Die Ausscheidung dieses schwach sauren wässerigen Harns hält an, solange die Einspritzung fortgesetzt wird (27 Stunden in einem Fall). Öfters, aber durchaus nicht immer, tritt eine Spur Eiweiss, die für eine kürzere oder längere Zeit anhält, im Urin auf. Bisweilen kommt eine vorübergehende Haematurie zum Vorschein, die ihren Ursprung aber immer, wie es scheint, in einer Verletzung der unteren Harnwege durch den Katheter hat. Dies wird dadurch bewiesen, dass die Haematurie in meinen Versuchen nur in 
katheterisierten Thieren zum Vorschein kam, die nach dem Tode leichte Blutungen in den unteren Harnwegen zeigten.

Zucker kommt ungefähr zwei Stunden nach dem Beginn der Einspritzung im Harn zum Vorschein. Wenn weniger als 75-100 cem einer $1 / 6$ molecularen NaCl-Lösung pro 15 Minuten eingespritzt werden, dauert es länger als zwei Stunden, bevor Zucker im Harn auftritt. Umgekehrt bringen grössere Mengen der Salzlösung den Zucker früher zum Vorschein. Doch sind die Thiere unter sich verschieden, indem manche Zucker früher im Harn ausscheiden als Andere. Die Glykosurie besteht 6-8 Stunden. Die Menge des ausgeschiedenen Zuckers ist zuerst klein, steigt aber rasch bis zu einem Maximum, um allmälig auf Null zu fallen. Wenn die Glykosurie ihren höchsten Punkt erreicht hat, kann die Concentration des Zuckers über $0,40 \%$ betragen. Diese Thatsache macht zuerst nicht den Eindruck einer schweren Glykosurie. Die Beträchtlichkeit der Zuckerausscheidung kommt aber gleich zum Vorschein, wenn daran erinnert wird, dass die Einspritzung der grossen Mengen Wasser die Menge des ausgeschiedenen Harns in der Zeiteinheit ungeheuer steigert. In diesen Versuchen mit beispielsweise $1 / 6$ molecularer NaCl-Lösungen secernieren die Kaninchen in ein oder zwei Stunden ungefähr soviel Harn wie sonst in 24. Dies besagt, dass, falls der Harn nicht durch die intravenöse Einspritzung von viel Wasser verdünnt würde, die Menge des ausgeschiedenen Zuckers zu Zeiten über $6 \%$ liegen würde, ein höchst schwerer Diabetes.

Weiter unten (S. 13 ff.) werden Fxperimente mitgeteilt, in welchen durch passende Versuchsanordnung die Zuckerausscheidung ohne eine bedentende Zunahme in der Menge des ausgeschiedenen Urinwassers zustande kam. In einen dieser Versuche stieg der Procentsatz Zucker im Urin bis auf $7,3 \%$.

In den soeben beschriebenen Versuchen wurde eine $1 / 6$ moleculare Kochsalzlösung zur Einspritzung benutzt. Ich wollte nun zunächst feststellen, welchen Einfluss Lösungen desselben Salzes in Concentrationen, die niedriger oder höher sind als diese, haben würden. Zwölf Kaninchen, die aus drei Würfen stammten, und die möglichst dasselbe Gewicht sowie für mehrere Tage dieselbe Kost genossen hatten, wurden dieselben Mengen ( $85 \mathrm{ccm}$ ), aber verschieden concentrierter Kochsalzlösungen, in der Zeiteinheit (15 Minuten) ein- 
gespritzt. Die Resultate dieser Experimente werden in folgenden Paragraphen kurz zusammengefasst.

a) Vier Versuche wurden mit $1 / 8$ molecularer Kochsalzlösung angestellt. Die Einspritzungen wurden $4-6$ Stunden fortgesetzt, Bei keinem der Kaninchen kam Zucker im Harn zum Vorschein.

b) Vier Versuche wurden mit 1/7 molecularer Kochsalzlösung angestellt. In drei dieser Experimente trat kein Zucker im Harn auf, obwohl die Einspritzungen 7 Stunden fortgesetzt wurden. In dem vierten Versuch dieser Reihe kam eine Spur Zucker nach 4 Stunden lang fortgesetzter Einspritzung zum Vorschein. Diese Spur verschwand aber sofort wieder und trat nicht wieder auf, obwohl ich mit der Einflössung 2 Stunden länger fortfuhr.

c) Die intravenöse Einspritzung von einer $2 / 18$ molecularen NaCl-Lösung rief eine Glykosurie in 2 Stunden 10 Minuten hervor. Nur eine Spur Zucker trat aber im Urin auf, und diese verschwand nach $1^{1 / 4}$ Stunde, obwohl die Einspritzung fortgesetzt wurde.

d) Eine $1 / 6$ moleculare NaCl-Lösung brachte eine Zuckerausscheidung in $1^{3 / 4}$ Stunden zum Vorschein. Die Spur Zucker, die zu dieser Zeit auftrat, stieg innerhalb 50 Minuten bis auf $0,15 \%$. Nachdem die Zuckerabgabe in dieser Stärke für 2 Stunden angedauert hatte, wurde der Versuch aufgegeben.

e) Eine $\% / 11$ moleculare NaCl-Lösung brachte eine Glyoksurie in 67 Minuten hervor. 22 Minuten später war die Concentration des Zuckers im Harn 0,12\%. Nachdem die Zuckerausscheidung 1 Stunde länger angehalten hatte, wurde der Versuch beendet.

f) Eine $1 / 5$ moleculare NaCl-Lösung brachte Zucker nach 22 Minuten zum Vorschein, 30 Minuten später enthielt der Urin $0,11 \%$ und 1 Stunde später $0,21 \%$ Zucker. Nach 2stündiger fortgesetzter Zuckerausscheidung wurde das Thier vom Operationstische entfernt.

Diese Versuche zeigen, dass Zucker um so früher im Harn zum Vorsehein kommt und im Allgemeinen, in um so grösseren Mengen erscheint, je höher die Concentration der eingespritzten Salzlösung ist. Unten werden noch weitere Beweise für die Richtigkeit dieses Satzes gebracht werden; da diese Versuche aber etwas verschieden sind von den soeben beschriebenen, will ich sie an dieser Stelle nicht anführen. 


\section{Ueber die Hervorrufung von Glykosurie durch andere Natriumsalze.}

Es ergab sich nun die Frage, ob das Vermögen von Kochsalz, eine Glykosurie hervorzurufen, eine Eigenschaft aller Natriumsalze ist. Die Thatsache, dass $\mathrm{K} u ̈ \mathrm{lz}$ eine Glykosurie durch die Einspritzung von essigsaurem-, kohlensaurem- und valeriansaurem Natrium hervorbringen konnte, sprach hierfür; dass es ihm aber andererseits nicht gelang, durch Natriumbromid und Natriumiodid Zucker im Harn zum Vorschein zu bringen, sprach gegen diese Idee. Da nun $\mathrm{K} u ̈ l z$ aber nur Lösungen brauchte, die den gleichen Procentsatz Salz enthielten (z. B. 1\%ige Lösungen von brom- und jodsaurem Natrium), anstatt äquimoleculare, erschien es möglich, dass das Misslingen der Versuche mit den zuletzt genannten Salzen darauf beruhte, dass seine Lösungen nicht genügend concentrirt waren. Ich unternahm es deshalb, eine Anzahl Versuche mit $\mathrm{NaBr}$, NaI und auch $\mathrm{NaNO}_{3}$ anzustellen. Wenn $75-100 \mathrm{cem}$ einer ${ }^{1 / 6} \mathrm{~mole}-$ cularen Lösung dieser Salze pro $15 \mathrm{Minuten}$ intravenös eingespritzt werden, kommt eine Glykosurie ebenso schnell und in derselben Weise zum Vorschein wie nach der Einspritzung einer äquimolecularen Kochsalzlösung.

\section{Das Vermögen von Calciumchlorid, die durch Natrinmsalz hervorgerufene Glykosurie zu hemmen.}

Es gelang mir in einer Anzahl von Versuchen den Beweis zu erbringen, dass die Glykosurie, die durch die Einspritzung einer reinen Natriumchloridlösung hervorgerufen wird, durch den Zusatzeines löslichen Calciumsalzes zu der Kochsalzlösung gehemmt wird. Wenn ungefähr 75-100 ccm einer $1 / 6$ molecularen NaCl-Lösung pro 15 Minuteu in die Bluthahn eines Kaninchens eingeflösst werlen, kommt innerhalb 2 Stunden Zucker im Urin zum Vorschein. Diese Glykosurie kann gehemmt werden, selbst wenn dieselbe Menge Flüssigkeit in der Zeiteinheit eingeflösst wird, wenn die reine Kochsalzlösung durch die folgende Lösung ersetzt wird: $25 \mathrm{ccm}{ }^{3} / \mathrm{s} \mathrm{moleculares} \mathrm{CaCl}_{2}+975 \mathrm{ccm}{ }^{1 / 6} \mathrm{~mole}-$ culares NaCl. Nachdem die Zuckerausscheidung hier- 
durch aufgehoben ist, kann sie wieder von Neuem hervorgerufen werden durch Rückkehr zu der reinen 1/6 molecularen Kochsalzlösung. An kräftigen, gesunden Kaninchen kann dies mehrmals hintereinander gethan werden. Durch passende Behandlung der Quetschhähne des in Fig. 1 abgebildeten Apparates kann von der Einspritzung der einen Lösung zur Einspritzung der anderen übergegangen werden, ohne das Thier auf irgend eine Weise zu stören.

Die specifischen Wirkungen der verschiedenen Salze zeigen sich erst nach einer ziemlich grossen Latenzzeit. Nachdem die Zuckerausscheidung durch eine reine $\mathrm{NaCl-Lösung} \mathrm{hervorgerufen} \mathrm{ist,} \mathrm{hört} \mathrm{sie} \mathrm{nicht} \mathrm{wieder} \mathrm{auf,} \mathrm{bis} \mathrm{die}$ Einspritzung der $975 \mathrm{~cm}^{1 / 6}$ molecularen $\mathrm{NaCl}+25 \mathrm{ccm}^{3} / 8$ molecularen $\mathrm{CaCl}_{2}$-Lösung $1-2$ Stunden gedauert hat. Nachdem die Zuckerausscheidung hierdurch aufgehoben ist, kann sie nicht wieder unter 1-2 Stunden von Neuem durch Rückkehr zu der reinen $\mathrm{NaCl}-L$ ösung hervorgerufen werden.

Das in dem vorhergehenden Paragraphen Gesagte gilt anscheinend auch für die Glykosurie, die durch reine $\mathrm{NaBr}-$, NaI- und $\mathrm{NaNO}_{3}$-Lösungen hervorgerufen wird. Es ist mir gelungen, die Zuckerausscheidung, die durch reine $1 / 6$ moleculare Lösungen dieser Salze hervorgerufen wird, aufzubeben, lange bevor die Menge des ausgeschiedenen Zuckers gleich der Menge des im Körper enthaltenen und auf diese Weise ausspülbaren Zuckers gleich war, indem die reinen Salzlösungen durch Mischungen von $975 \mathrm{ccm}$ der $1 / 6$ molecularen Natriumsalzlösungen mit $25 \mathrm{ccm} 3 / 8$ molecularen $\mathrm{CaCl}_{2}-$ Lösungen ersetzt wurden. In all meinen bisher angestellten Versuchen haben aber die giftig wirkenden Anionen dieser Natriumsalze das Thier getödtet, bevor ich Zucker ein zweites Mal im Harn bekommen konnte.

Der folgende Versuch mag dazu dienen, das Vorhergesagte etwas zu erläutern. Das Experiment zeigt, wie Glykosurie in einem Kaninchen durch eine reine Kochsalzlösung hervorgerufen werden kann, und wie diese Glykosurie durch Zusatz eines kleinen Betrages Calciumchlorid zur Kochsalzlösung aufgehoben wird, um von Neuem hervorgerufen zu werden durch Rückkehr zu der reinen Natriumchlorid-Lösung.

Die Zeit ist in der ersten Spalte angegeben. Die zweite Spalte zeigt wie viel Salzlösung in den vergangenen (gewöhnlich den 
letzten 15) Minuten eingespritzt worden ist. Die Menge des ausgeschiedenen Harns in derselben Zeiteinheit ist in der dritten Spalte angegeben. Der aufrechtstehende Pfeil $(\uparrow)$ bedeutet, dass der Procentsatz Zucker seit der letzten Analyse gestiegen ist; der umgekehrte Pfeil $(\downarrow)$, dass der Procentsatz Zucker gefallen ist. Kein Pfeil meint, dass die Menge Zucker ungeändert geblieben ist. Das Experiment ist mit allen Details wiedergegeben, um zu zeigen, wie all die Versuche, auf welchen sich die hier mitgeteilten Resultate stuitzen, ausgefübrt wurden.

\section{Protokoll A.}

Grosses, männliches Kaninchen. $2700 \mathrm{~g}$. Für mehrere Tage mit Alfafaheu, Gerste und Mohrrüben gefüttert. Harnblase durch Katheterisation entleert.

Injectionsflüssigkeit: 1/6 moleculares $\mathrm{NaCl}$ abwechselnd mit $975 \mathrm{ccm}$ $1 / 6$ moleculares $\mathrm{NaCl}+25 \mathrm{~cm} \mathrm{3/8}$ moleculares $\mathrm{CaCl}_{2}$.

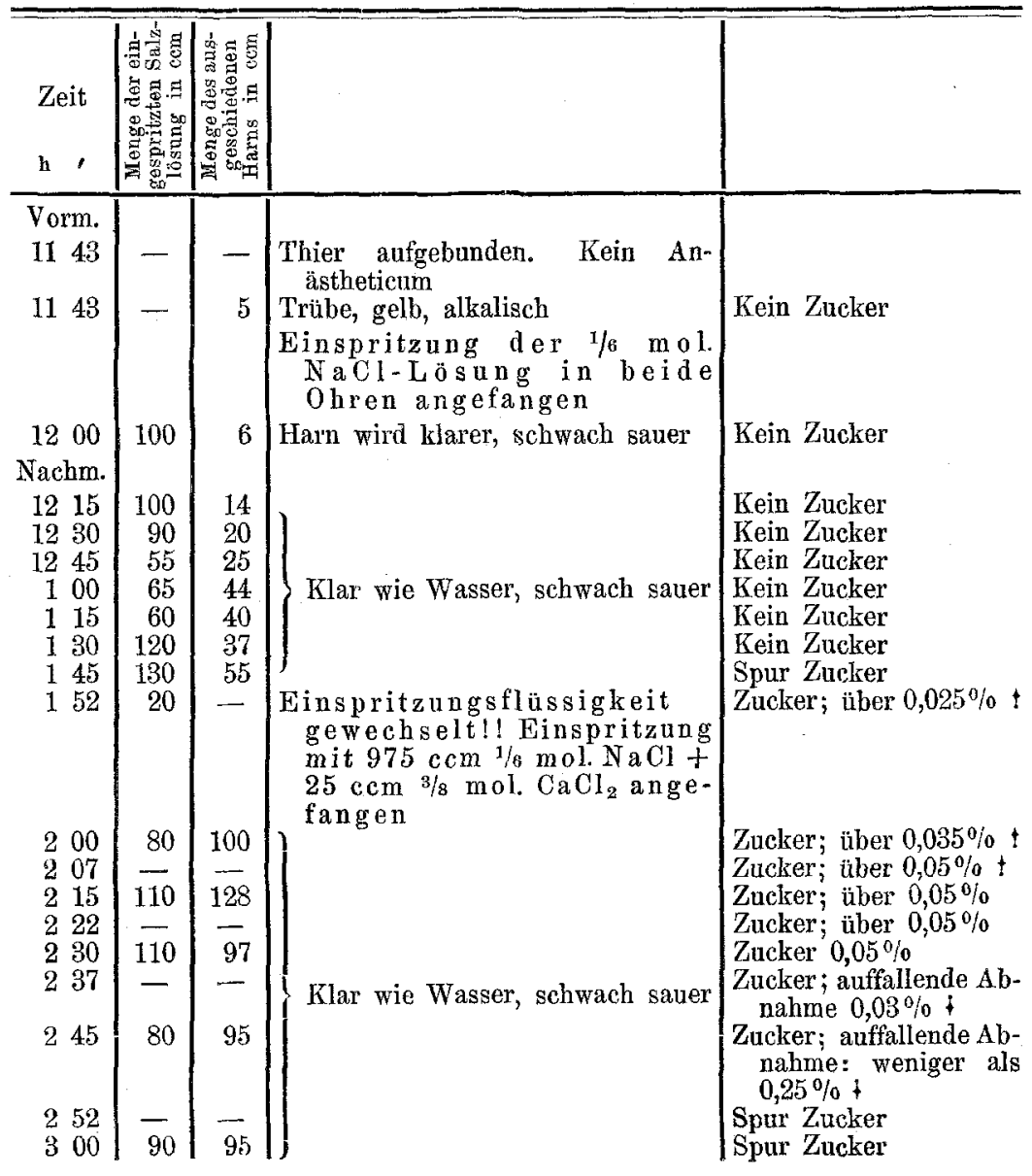


Ueber die Hervorrufung a. Hemmung von Glykosurie in Kaninchen etc. 11

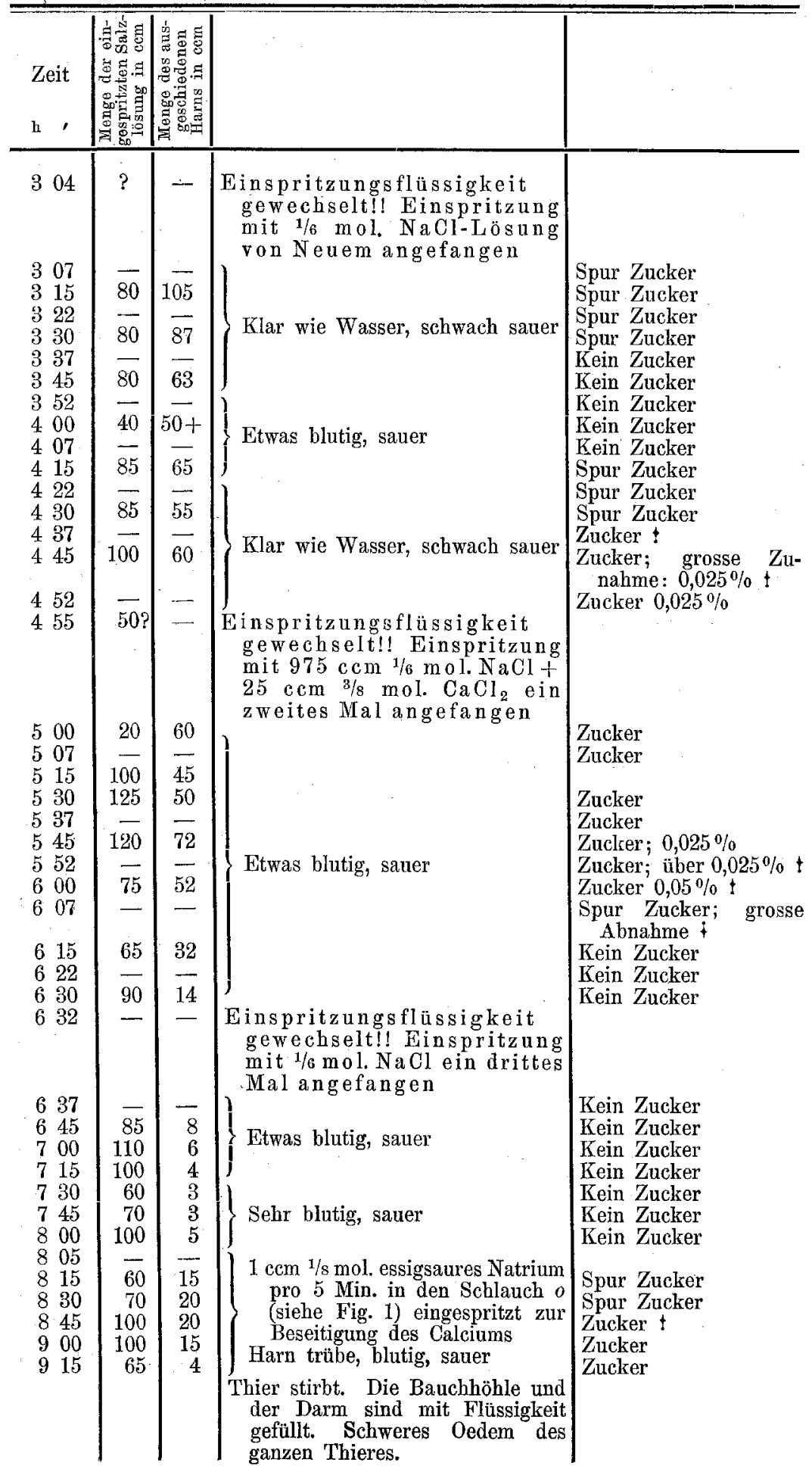


Ich habe Protokolle von sieben Versuchen, in welchen eine Zuckerausscheidung durch eine reine Kochsalzlösung hervorgerufen wurde, durch eine Mischung von derselben Kochsalzlösung mit Calciumchlorid beseitigt wurde, um wiederum durch reine Kochsalzlösung hervorgerufen zu werden. In den verschiedenen Versuchen konnte dies ein- bis dreimal wiederholt werden.

Aber nicht in allen Versuchen konnte ich die Kochsalzglykosurie durch Zufügung von Calciumchlorid beseitigen. In drei Versuchen, die unter den obigen nicht mitgezählt sind, gelang es mir nicht die Zuckerausscheidung aufzuheben. Was in diesen Fällen die Ursache der uncontrollirbaren Zuckerausscheidung war, kann nicht mit Bestimmtheit gesagt werden. Ein Thier hatte nach einer Verletzung eine Morphiumeinspritzung bekommen, welche an und für sich Glykosurie in Kaninchen hervorrufen kann. Bei einem zweiten Kaninchen wurde eine schwere Glykosurie (über $0,1 \%$ ) durch Finspritzung einer $1 / 6$ molecularen Kochsalzlösung hervorgerufen. Die Zuckermenge wurde bis auf eine Spur beseitigt durch eine Mischung von $\mathrm{NaCl}$ und $\mathrm{CaCl}_{2}$. Ich bekam Zucker über $0,1 \%$ ein zweites Mal durch Rückkehr zu der reinen Kochsalzlösung. Diese Zuckerausscheidung wurde wieder bis zu $0,025 \%$ herabgesetzt durch Einspritzung der $\mathrm{NaCl}$-CaCla-Lösung. Die Zuckerausscheidung stieg sodann ein drittes Mal, obwohl die Einspritzung mit dem $\mathrm{NaCl}-\mathrm{CaCl}_{2}-\mathrm{Gemisch}$ fortgesetzt wurde. Im dritten Fall bekam ich eine schwere Glykosurie nach Einspritzung von $100-110 \mathrm{ccm}$ pro 15 Minuten einer $1 / 6$ molecularen NaClLösung. Die Zuckerausscheidung wurde durchaus nicht durch Zugabe von $\mathrm{CaCl}_{2}$ zur reinen Kochsalzlösung beeinflusst. Weiter unten werden noch andere Versuche erwähnt werden, in welchen durch Einspritzung von zu grossen Mengen oder von zu concentrirten $\mathrm{NaCl}$ - Lösungen uncontrolirbare Glykosurien zu Stande kamen. Früher suchte ich die Erklärung dieser Glykosurien hauptsächlich in gewissen äusseren mitwirkenden Umständen. Ich glaube aber jetzt, dass mit Ausnahme des Versuches, in welchem Morphium eingespritzt wurde, welches an und für sich eine Glykosurie im Kaninchen hervorrufen kann, dass die Ursache dieser uncontrolirbaren Glykosurien in einer dauernden Verletzung der Medulla oblongata liegt. Wie weiter unten gezeigt werden soll, liegt der Angriffspunkt aller dieser Salze insoweit als sie eine Glykosurie hervorrufen können in einer Einwirkung auf die Medulla. Das Anbinden der Thiere allein bringt eine Glykosurie bei Kaninchen nicht zu Stande, obwohl dies 
bei Katzen möglich zu sein seheint, bei denen Böhm und Hoffman ${ }^{1}$ ) regelmässig Zucker im Harn fanden, wenn die Thiere auf das Operationsbrett gebunden waren. Bei sechs Kaninchen, die ich auf den Tisch band und hier 24-36 Stunden liess, kam Zucker nicht einmal im Harn zum Vorschein.

\section{Versuche zur Localisirung des Angriffspunkts der Salz- lösungen.}

Es ist durch die Versuche von $K \ddot{l} l z$ gezeigt worden, dass die Glykosurie, die dureh Einspritzung reiner Kochsalzlösungen in Kaninchen hervorgerufen wird, nicht mehr zum Vorschein kommt, wenn die Nervi splanchnici durchschnitten sind, und dass eine vorhandene Glykosurie nach dieser Operation aufhört. Diese Thatsachen scheinen darauf hinzuweisen, dass der Diabetes nervösen Ursprungs ist, und dass das Kochsalz auf das sogenannte Diabetescentrum der Medulla oblongata wirkt. Um diese Idee etwas weiter zu prüfen, machte ich eine Anzahl Experimente, in welchen der Versuch gemacht wurde, die Wirkung des Kochsalzes soweit wie möglich zu localisiren. Falls die verschiedenen Salzlösungen durch Einwirkung auf die Medulla eine Zuckerausscheidung zu Stande bringen, war es zu erwarten, dass, wenn die Einspritzungen möglichst direct in die Blutgefässe der Medulla gemacht würden, die Zuckerausscheidung einen höheren Grad erreichen würde, als wenn dieselbe Menge derselben Salzlösung in ein peripherisches Blutgefäss eingeführt würde. Um vergleichbare Resultate zu bekommen wurden eine Reihe Versuche angestellt, in welchen immer möglichst gleich grosse, gleich alte und gleich ernährte Kaninchen so behandelt wurden, dass das eine Kaninchen eine Einspritzung in das peripherische Ende der A. femoralis, das andere in das centrale Ende der abgebundenen A. axillaris bekam.

Da ich in diesen Versuchen concentrirtere und deshalb viel geringere Mengen Salzlösung einspritzte als in den bisher beschriebenen Experimenten, konnte ich den in Figur 2 abgebildeten Einspritzungsapparat besser gebrauchen als den früher beschriebenen. Weiterhin war es in den folgenden Experimenten eine nothwendige Bedingung,

1) Böhm und Hoffmann. Citirt nach Naunyn, Der Diabetes melitus. Wien 1898. 
dass die Mengen der eingespritzten Salzlösungen g e na u regulierbar sind, um vergleichbare Resultate zu bekommen. Der Apparat, welcher in Figur 2 abgebildet ist, erlaubt dies vollständig. $C$ ist eine gewöhnliche Pravazspritze, die durch das verstellbare Ventil $V$ mit den Gummischläuchen $A$ und $B$ in Verbindung steht. Das freie Ende von $A$ taucht

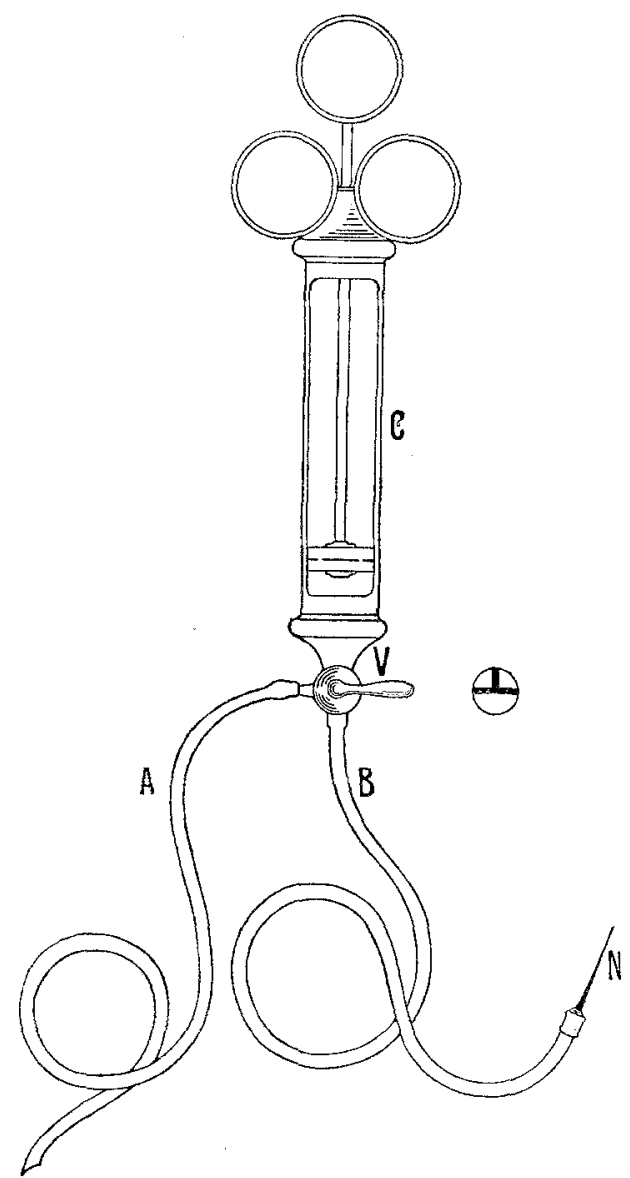

Fig. 2. in ein Becherglas, welches die erwärmte, zur Einspritzung dienende Lösung enthält, während $B$ mit der hypodermischen Nadel $N$, die in die Blutbahn des Thieres eingeführt wird, versehen ist. Durch passende Einstellung des Ventils und durch Hebung und Senkung des Kolbens der Spritze kann irgend eine Flüssigkeit schnell durch den Schlauch $A$ in den Cylinder $C$ eingesogen werden, um mit beliebiger Geschwindigkeit durch den Schlauch $B$ in das Thier eingespritzt zu werden.

Um eine möglichst directe Wirkung auf die Medulla oblongata zu bekommen, versuchte ich, die Salzlösungen so einzuspritzen, dass sie durch die A. vertebralis der einen Seite in die Medulla direct eindrangen. Indessen ist diese Arterie so klein, dass die hypodermische Nadel nicht direct in sie eingeführt werden kann. Die Einspritzungen wurden deshalb centralwärts in die A. axillaris gemacht nach Abbindung derselben, um eine peripherische Fortbewegung der Salzlösung zu vermeiden. Manchmal wurde, um den Zutritt der Salzlösung in die Medulla noch weiter zu versichern, auch die $A$. carotis abgebunden. 
Die notwendige Operation zur Freilegung der verschiedenen Blutgefässe wurde folgenderweise ausgeführt. Das Fell des Kaninchens wurde nach einem Hautschnitt in der Mittellinie reflectirt, und die Mm. Pectorales der rechten Seite durch ein en Schnitt quer geteilt. Blutungen wurden durch Haemostaten leicht gestillt und ein wenig sorgfältiges Seciren legte das obere Ende der A. axillaris, die A. subclavia und das untere Ende der A. carotis mit den darüber liegenden Nerven frei. Die Blutgefässe wurden vorsichtig von den Nerven getrennt und die zur Abbindung dienenden Seidenfäden mittelst einer gebogenen Nadel um die Arterien gezogen.

Ich möchte betonen, dass die Thiere nie ein Anestheticum bekamen, da die Natur der Versuche dies nicht erlaubt. Die ganze Operation kann in vier Minuten vollendet werden, und wenn nur ein scharfes Messer gebraucht wird, bewegt sich das Thiernichteinmal; das Kaninchen leidet scheinbar gar nicht. Um sicher zu sein, dass die Operation an und für sich nicht zu einer Glykosurie führt, habe ich mehrere Versuchsthiere als Controlle 2-10 Stunden auf dem Tisch liegen lassen und den Harn sorgfältig auf Zucker analysirt. In keinem der Thiere kam Zucker zum Vorschein. Ich glaube deshalb, dass wir mit Sicherheit sagen können, dass die nothwendige Operation nicht die Ursache einer Glykosurie ist. Verletzung der Nerven durch Durchschneidung, Ziehen, Austrocknen und dergl. wurden auf das Peinlichste vermieden.

Wie schon in meinen früheren Mittheilungen hervorgehoben ist, ist die Leichtigkeit, mit welcher eine Glykosurie in verschiedenen, gleich grossen, gleich alten und gleich gefütterten Kaninchen hervorgerufen werden kann, verschieden. Es ist deshalb nothwendig, eine grössere Zahl Versuche anzustellen, um vergleichbare Resultate zu bekommen. Ich habe im Ganzen sechs Versuchsreihen gemacht, in welchen 14 Kaninchen in der oben beschriebenen Weise benutzt wurden. In diesen Versuchen stellte es sich nun heraus, dass die Zuckerausscheidung etwas früher anfängt, und dass der Betrag ausgeschiedenen Zuckers grösser ist, wenn eine $1 / 2$ oder $1 / 1$ moleculare $\mathrm{NaCl}-\mathrm{Lösung}$ centralwärts in die abgebundene A. axillaris eingespritzt wird, als wenn dieselbe Menge derselben Salzlösung in ein peripherisches Blutgefäss eingeflösst wird. Wenn die concentrirte Salzlösung in die A. axillaris eingespritzt wird, genügen 
schon 25-30 cem, um den Anstoss zu einer Zuckerausscheidung zu geben. Wie in den schon oben beschriebenen Versuchen (S. 6) hält bei fortgesetzter Einspritzung auch hier die Zuckerausscheidung solange an, bis all der auf diese Weise ausscheidbare Zucker secernirt ist. Wenn die Einspritzung aber aufgehoben wird, bevor dieser Punkt erreicht ist, so hört die Glykosurie gewöhnlich in 30-40 Minuten auch auf. Wie schon oben hervorgehoben ist, ist dies beinahe ausnahmslos der Fall, wenn die concentrirten Kochsalzlösungen in ein peripherisches Blutgefäss eingeflösst sind, und wenn der Versuch nicht zu lange gedauert hat. Falls aber die Einspritzung centralwärts in die A. axillaris gemacht ist, passirt es sehr oft, dass die Zuckerausscheidung nicht aufhört, sondern 2-6, sogar 8 Stunden anhält. Insoweit als wir die Natur der Einwirkung des Kochsalzes (und der noch weiter unten beschriebenen Salze) auf die Medulla noch nicht kennen, können wir einstweilen nur sagen, dass die Kochsalzlösung die Medulla tiefer schädigt, wenn sie in die A. axillaris, als wenn sie in ein peripherisches Blutgefäss eingespritzt wird. Diese tiefere Einwirkung ist nichts Anderes als ein Ausdruck der höheren Concentration, in welcher die Salzlösung die Medulla trifft, wenn sie in die A. axillaris eingespritzt wird, als wenn dieselbe Menge derselben Salzlösung in ein peripherisehes Blutgefäss, wo eine freiere Mischung mit dem Blut stattfinden kann, eingeführt wird.

Beiläufig möchte ich sieben Experimente erwähnen, in welchen ich versuchte, die durch Einspritzung einer $1 / 2$ oder $1 / 1$ molecularen NaCl-Lösung in die A. axillaris hervorgerufene Glykosurie durch Zusatz von $\mathrm{CaCl}_{2}$ aufzuheben, indessen ohne Erfolg, selbst wenn diejenige Menge $\mathrm{CaCl}_{2}$ mit Berücksichtigung der entsprechenden Concentration des Natriumchlorids eingespritzt wurde, die sich bei Verwendung einer $1 / 6$ molecularen $\mathrm{NaCl}$-Lösung als wirksam erwiesen hatte. Die durch $1 / 2$ oder $1 / 1$ moleculare CaCl-Lösung herbeigeführte Glykosurie hielt nichtsdestoweniger an, ohne selbst eine Verminderung in der Menge des ausgeschiedenen Zuckers hervorzurufen. Selbst wenn eine Mischung von $1 / 2$ oder $1 / 1$ moleculare $\mathrm{NaCl}-\mathrm{Lösung}$ mit $\mathrm{CaCl}_{2}$ in ein gesundes Kaninchen eingespritzt wurde, kam eine Glykosurie zum Vorschein in derselben Weise, als wenn eine reine Kochsalzlösung eingespritzt worden wäre. Die Erklärung dieser Ergebnisse ist vielleicht in der ungleichen Diffusionsgeschwindigkeit der zwei Salze zu suchen. 
Die Menge des ausgeschiedenen Harns hängt ron der Menge der eingeflössten Salzlösung und der Concentration derselben $a b^{1}$ ). Wenn nur wenig Kubikcentimeter (20-25) einer concentrirten Salzlösung eingesprizt werden - was der Fall ist, wenn die Einspritzung in die A. axillaris gemacht wird - ist die Harnsecretion gar nicht oder nur wenig gesteigert. Umgekehrt ist eine grosse Harnsecretion die Folge der Einflössung einer grösseren Menge der Salzlösung. Der Procentsatz Zucker, d. h. die Concentration des Zuckers im Harn, ist sehr variabel, und zwar läuft sie nicht der Menge des ausgeschiedenen Harns proportional, eher umgekehrt. Wenn die Menge des secernirten Harns klein ist, mag der Procentsatz Zucker zu Zeiten bis auf $7,3 \%$ steigen. Wenn die Harnsecretion hoch ist, mag nur eine Spur Zucker vorhanden sein. Massgebend für die Schwere des Diabetes ist die Menge des ausgeschiedenen Zuckers worin beide diese Factoren berücksichtigt werden. In den 2-6 Stunden anhaltenden Glykosurien mögen 1-1,85 g Zucker im Harn ausgeschieden werden, woraus ohne weiteres hervorgeht, dass die Glykosurien die durch die Einspritzung concentrirter Kochsalz- (und anderer) Lösungen in die A. axillaris hervorgerufen werden den schwersten Formen des experimentellen Diabetes, die uns bekannt sind, gleichgestellt werden müssen.

Die Thatsachen, dass erstens der Procentsatz Zucker im Harn sehr variabel und nicht der Menge des abgesonderten Harns proportional ist, dass zweitens ein bedeutendes Quantum Zucker ohne eine Zunahme in der Urinausscheidung im Harn zum Vorschein kommen kann, und dass drittens eine schwere Polyurie olne eine Spur Zucker durch rerdünnte Kochsalzlösungen hervorgerufen werden kann, sind von Wichtigkeit insoweit, als $\mathrm{Jacobj}^{2}$ ) und andere die Ursache der Zuckerausscheidung nach der Einspritzung verschiedener Diuretica (einschliesslich reiner Kochsalzlösungen) in der gesteigerten Harnsecretion sehen wollen. Nach Jacobj muss folglich der Kochsalzdiabetes als ein Diabetes, der durch Änderungen in den Nieren hervorgerufen wird - als ein Diabetes renalis - angesehen werden. Ueber die Ursache der Zuckerausseheidung infolge der Eingabe diuretischer Mittel im Allgemeinen will ich mich hier nicht äussern.

1) Für Details siehe meine zweite Mittheilung über Glykosurie, l. c. S. 107.

2) Jacobj, Arch. f. exper. Pathol. u. Pharmak. Bd. 35 S. 213. 1895.

E. Pflüger, Archiv für Physiologie. Bd. 109. 
Der Diabetes, der durch Natrium Chlorid und die noch weiter unten erwähnten Salze hervorgerufen wird, ist aber sicher nicht ein Diabetes, dessen Ursache in einer gesteigerten Harnsecretion liegt. Wäre Jacabj's Ansicht die richtige, so müsste die Zuckerausscheidung desto schwerer sein, je höher die Diurese ist, ein Umstand, der in meinen Versuchen durchaus nicht der Fall ist. Hierin liegt ein weiterer Beweiss für die nervöse Natur des Kochsalzdiabetes. Di e Kochsalzeinflössung bringt Änderungea in zwei Organen hervor. Durch ihre Wirkung auf die Niere steigert sie die Harnsecretion; durch ihre Wirkung auf die Medulla oblongata kommt es zu einer Zuckerausscheidung, dessen Menge der Einwirkung des Kochsalzes proportional ist. In dieser Weise bekommen wir eine ungezwungene und einfache Erklärung der experimentellen Ergebnisse.

\section{Ueber andere (nicht Natrinm-)Salze, die eine Glykosurie hervorrufen können.}

Das oben beschriebene Verfahren der Jangsamen Einspritzung ron Salzlösungen in das centrale Ende der abgebundenen A. axillaris erlaubte mir die Wirkung von verschiedenen Salzlösungen zu untersuchen, welche bei Einspritzung in die Ohrrenen schnell tödtlich wirken. Der Tod ist wahrscheinlich durch eine giftige Wirkung dieser Salze auf das Herz bedingt, da Herzstillstand und die Symptome der Asphyxie dem Tod vorhergehen. Wenn nun die Salzlösungen in die A. axillaris eingespritzt werden, wirken sie zunächst auf die Medulla oblongata, werden aber, ehe sie das Herz erreichen, so durch das Blut verdünnt, dass sie das Leben des Thieres nicht mehr bedrohen.

Es wurden zuerst eine Anzahl einwerthige Salze gebraucht, $\mathrm{LiCl}, \mathrm{KCl}, \mathrm{NH}_{4} \mathrm{Cl}$ und die schon erwähnten benutzbaren Natriumsalze. Alle die Natriumsalzlösungen, welche sich früher als wirksam herausgestellt hatten, wenn sie in die Ohrvenen eingespritzt wurden, erwiesen sich als noch wirksamer in Bezug auf die Hervorrufung einer Glykosurie, wenn sie centralwärts in die abgebundene A. axillaris eingespritzt wurden.

$\mathrm{NH}_{4} \mathrm{Cl}$ stellte sich als wirkungslos in dieser Hinsicht heraus, selbst wenn in der Zeiteinheit viel grössere Quanti- 
täten einer äquimolecularen Lösung eingespritzt wurden, als in den Versuchen mit Natriumsalzen. Eine Hämoglobinurie (wahrscheinlich durch eine Hämolyse der rothen Blutkörperchen hervorgerufen) entwickelt sich stets kurz nach dem Beginn der $\mathrm{NH}_{4} \mathrm{Cl}$-Einspritzung, und fäbrt fort bis das Thier unter den Symptomen der Erstickung zu Grunde geht. Während dieser ganzen Zeit, die eine Stunde überschreiten mag, kommt Zucker nicht ein Mal im Harn zum Vorsehein.

LiCl- und $\mathrm{KCl}$-Lösungen bringen regelmässig und ebenso leicht eine Glykosurie hervor wie NaCl. Nur müssen die Einspritzungen vorsichtig gemacht werden, so dass die Kaninchen nicht bei einer zu hohen Concentration dieser Salze im Blute sterben. Die folgenden abgekürzten Protokolle mögen als Beispiele dienen, um die Hervorrufung des Diabetes durch diese Salze zu erläutern.

\section{Protokoll B.}

Granes, männliches Kaninchen. 1500 g. Für mehrere Tage mit Alfalfa, Heu, Gerste und die letzte Nacht mit Mohrrüben gefüttert. Harnblase durch Katheterisation entleert.

Injektionsflüssigkeit: 1/2 moleculare KCl-Lösung.

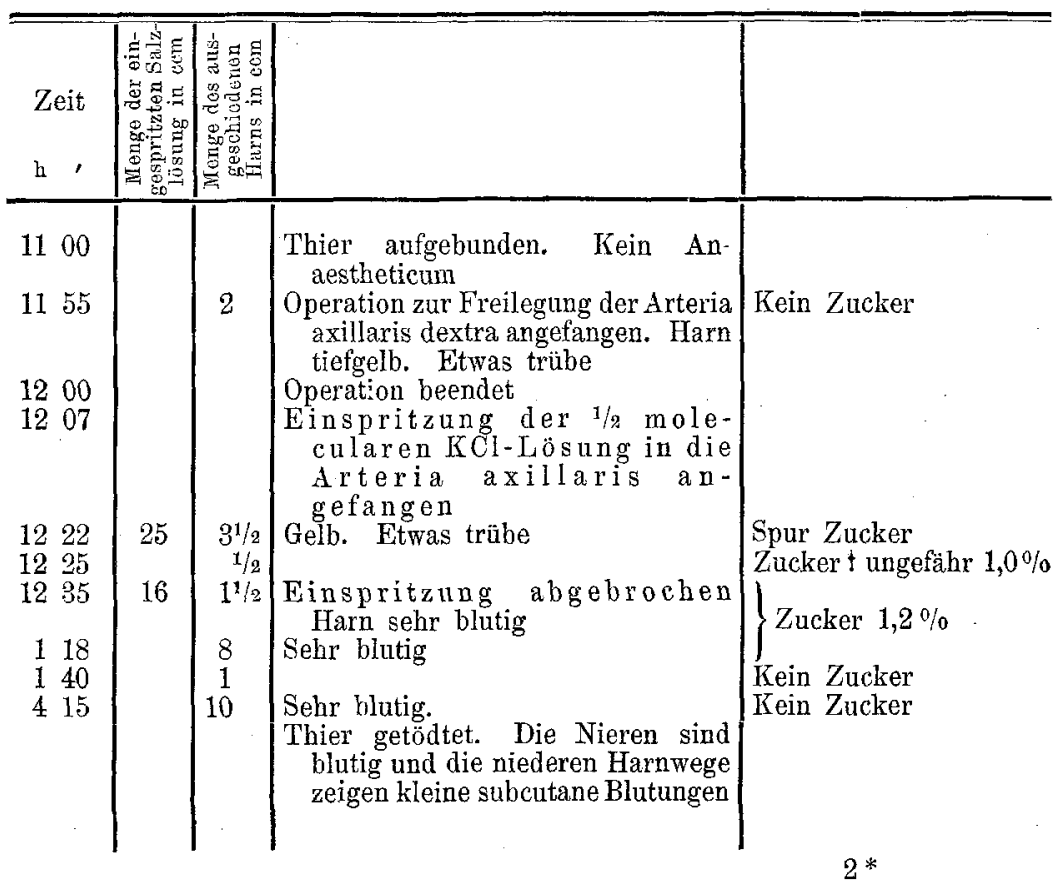




\section{Protokoll C.}

Graues, männliches Kaninchen. $1150 \mathrm{~g}$. Heute nicht gefüttert. Harnblase durch Katheterisation entleert.

Injectionsflüssigkeit: 1/2 moleculare LiCl-Lösung.

\begin{tabular}{|c|c|c|c|c|}
\hline Zeit & 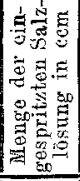 & 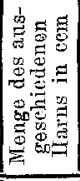 & & \\
\hline 230 & & & $\begin{array}{l}\text { Thier aufgebunden. Kein Anaesthe- } \\
\text { ticum }\end{array}$ & \\
\hline 310 & & 8 & Harn bräunlich, etwas trübe & Kein Zucker \\
\hline 337 & & 2 & $\begin{array}{l}\text { Operation zur Freilegung der Arteria } \\
\text { axillaris dextra angefangen. Harn } \\
\text { bräunlich, etwas trübe }\end{array}$ & Kein Zucker \\
\hline 341 & & & $\begin{array}{l}\text { Operation beendet. Thier wird un- } \\
\text { gestört sich selbst überlassen }\end{array}$ & \\
\hline 419 & & $3 / 4$ & $\begin{array}{l}\text { Harn bräunlich, etwas trübe } \\
\text { Einspritzung der } 1 / 2 \text { molecur } \\
\text { laren LiCl-Lösug in die } \\
\text { Arteria axillaris ange- } \\
\text { fangen }\end{array}$ & Kein Zucker \\
\hline 422 & 12 & 4 & Harn heller & Kein Zucker \\
\hline $\begin{array}{l}426 \\
432\end{array}$ & $\begin{array}{l}16 \\
24\end{array}$ & $\begin{array}{l}10 \\
15\end{array}$ & $\begin{array}{l}\text { Harn hell-gelb. Einspritzung } \\
\text { abgebrochen }\end{array}$ & Zucker $0,5 \%$ \\
\hline $\begin{array}{ll}4 & 36 \\
5 & 00 \\
5 & 18\end{array}$ & & $\begin{array}{r}17 \\
6 \\
4\end{array}$ & $\begin{array}{l}\text { Harn hell-gelb } \\
\text { Harn dunkel-gelb } \\
\text { Thier getödtet }\end{array}$ & $\begin{array}{l}\text { Zucker } 0,35 \% \\
\text { Kein Zucker } \\
\text { Kein Zucker }\end{array}$ \\
\hline
\end{tabular}

Sodann wurden eine Anzahl Experimente mit zweiwerthigen Salzen angestellt. Ich versuchte zuerst $\mathrm{ob} \mathrm{CaCl} \mathrm{Cl}_{2}$, welches die Glykosurie hemmt, wenn es einer reinen Kochsalzlösung von einer nicht zu bohen Concentration zugefügt wird, für sich allein eine Glykosurie hervorrufen würde. Nach Einspritzung reiner Chlorkalciumlösungen starben aber ausnahmslos alle Kaninchen an Herzstillstand, ehe Zucker im Urin zum Vorschein kam. $\mathrm{MgCl}_{2}$ wirkte in gleicher Weise tödtlich.

Sehr interessant aber ist die Thatsache, dass das dem Calciumchlorid chemisch sehr nahe stehende Strontiumchlorid eine schwere Zuckerausscheidung hervorrief, wenn nur wenige Cubikcentimeter einer $3 / 8$ molecularen Lösung in das centrale Ende der abgebundenen A. axillaris langsam eingespritzt wurden. Scheinbar genügt ein kleinerer chemisch äquivalenter Betrag $\mathrm{SrCl}_{2}$, um eine Glykosurie hervorzurufen als irgend 
ein anderes Salz. In den vier Versuchen, die mit $\mathrm{SrCl}_{2}$ angestellt wurden, dienten $11-20$ cem einer $3 / 8$ molecularen Lösung, um Zucker im Harn zum Vorschein zu bringen, während im Allgemeinen grössere Mengen concentrirterer Natriumsalzlösungen verlangt wurden, um dasselbe Ziel zu erreichen. In den Versuchen mit $\mathrm{SrCl}_{2}$ muss noch mehr darauf geachtet werden, dass man das Versuchsthier nicht durch eine zu rasche Einspritzung tödtet als bei $\mathrm{KCl}$ und $\mathrm{LiCl}$.

Das folgende Experiment zeigt, wie eine Glykosurie durch $\mathrm{SrCl}_{2}$ hervorgerufen werden kann.

\section{Protokoll D.}

Graues, männliches Kaninchen. 750 g. Während der letzten Woche mit Heu und Gerste gefüttert.

Injectionsflüssigkeit: ${ }^{3 / 8}$ moleculare $\mathrm{SrCl}_{2}$-Lösung.

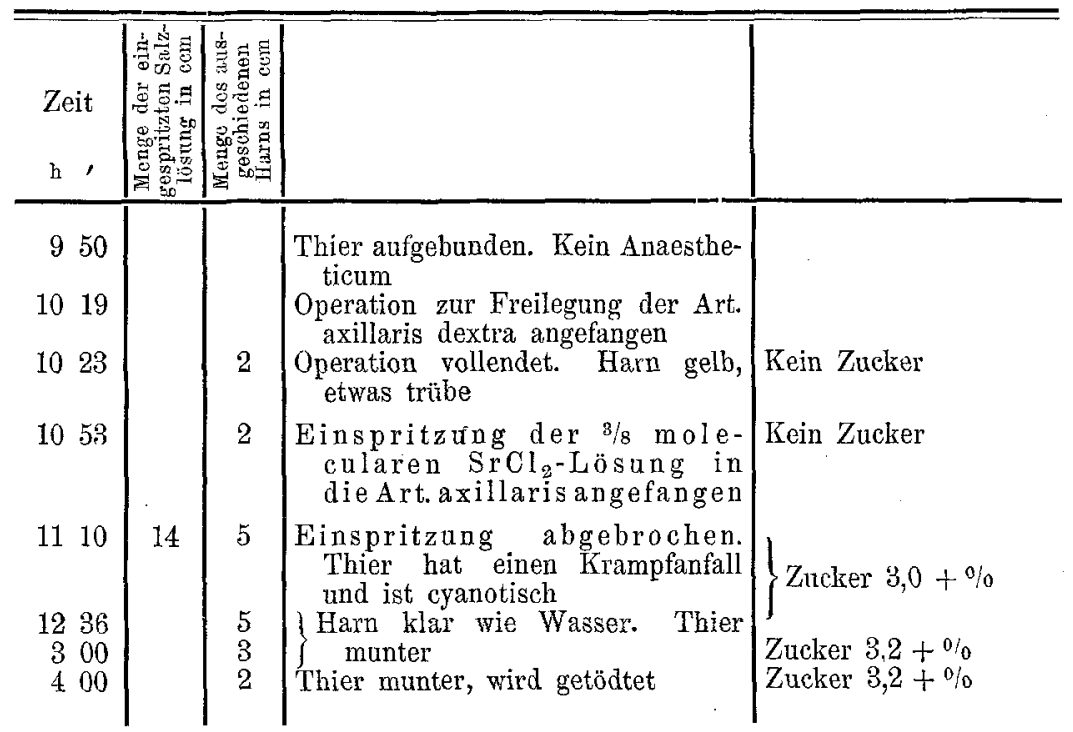

Da eine gewisse Möglichkeit vorhanden war, dass die Wirkung der verschiedenen Salzlösungen eine rein osmotische war, indem alle die wirksamen Salzlösungen eine böhere Concentration besassen als die osmotische Concentration des Kaninchenblutes, machte ich eine Anzahl Controlversuche mit Lösungen von Nicht-Elektrolyten, die denselben oder einen höheren osmotischen Druck hatten als die wirksamen Salzlösungen. Glycerin, Harnstoff und Aethylalkohol wurden in dieser Weise benutzt. Die Einspritzung von keiner dieser 
Lösungen aber brachte eine Glykosurie herbei, selbst wenn sie auf demselben Wege und in denselben oder grösseren Beträgen als die Lösungen der wirksamen Elektrolyte eingeführt wurden. Sobald aber die Einspritzung der Lösung des Nicbt-Elektrolyten durch die Finspritzung einer reinen NaCl-Lösung ersetzt wurde, kam Zucker in der gewöhnlichen Weise im Urin zum Vorschein.

Im Ganzen wurden sechs Versuche angestellt, zwei mit Glycerin, zwei mit Harnstoff und zwei mit Aethylalkohol. Es wird wohl genügen nur die abgekürzten Protokolle zwei dieser Experimente hier wiederzageben. Diese Versuche zeigen, dass die Glykosurie bervorrufenden Chemikalien nicht etwa durch eine Wasserentziehung, sondern durch einen specifisch chemischen oder physiko-chemischen Effect wirksam sind. Die Natur dieser Wirkung könnte einstweilen nur hypothetisch discutirt werden, was olne Nutzen ist.

\section{Protokoll E.}

Graues, weibliches Kaninchen (Belg. Hase). $1700 \mathrm{~g}$. Während der letzten Woche mit Heu, Gerste und Mohrrüben gefüttert.

Injectionsflüssigkeit: 1/1 moleculare Aethylalkobol-Lösung, später 1/2 moleculare NaCl-Lösung.

\begin{tabular}{|c|c|c|c|c|}
\hline $\begin{array}{l}\text { Zeit } \\
\mathrm{h},\end{array}$ & 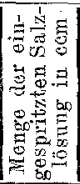 & 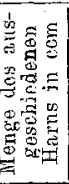 & & \\
\hline 415 & & & Thier aufgebunden. Kein Anaesthe- & \\
\hline 446 & & & Operation zur Freilegung der Arteria & \\
\hline $\begin{array}{l}450 \\
454\end{array}$ & & & $\begin{array}{l}\text { axillaris dextra angefangen } \\
\text { Operation vollendet } \\
\text { Einspritzung der } \mathbf{1}_{1} \text { molecu- } \\
\text { laren } \mathrm{C}_{8} \mathrm{H}_{5} \mathrm{OH}-\mathrm{Losung} \text { in } \\
\text { die Arteria axillaris an- } \\
\text { gefangen }\end{array}$ & \\
\hline 514 & 80 & & $\begin{array}{l}\text { Einspritzung abgebrochen. } \\
\text { Schwere Haemoglobinurie }\end{array}$ & Kein Zucker \\
\hline 525 & & $1 / 2$ & & Kein Zucker \\
\hline 552 & & $1 / 2$ & $\begin{array}{l}\text { Einspritzungsflussigkeit } \\
\text { gewechselt. Injection mit } \\
\text { 1/2 molecularer NaCl-Lö- } \\
\text { sungangefangen. }\end{array}$ & Kein Zucker \\
\hline 556 & 16 & $1 / 2$ & & Kein Zucker \\
\hline $\begin{array}{l}604 \\
608\end{array}$ & $\begin{array}{l}32 \\
16\end{array}$ & $\begin{array}{l}2 \\
3\end{array}$ & $\begin{array}{l}\text { Schwere Hämoglobinurie } \\
\text { Thier wird getödtet }\end{array}$ & $\begin{array}{l}\text { Spur Zucker } \\
\text { Zucker ungefähr } 0,21 \%\end{array}$ \\
\hline
\end{tabular}




\section{Protokoll F.}

Männliches Kaninchen (Belg. Hase). 1550 g. Während der letzten Woche mit Heu, Gerste und Mohrrüben gefüttert.

Injectionsflüs sigke it: $1 / 1$ moleculare Harnstofflösung, später $1 / 2$ moleculare NaCl-Lösung.

\begin{tabular}{|c|c|c|c|c|}
\hline Zeit & 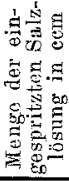 & 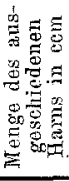 & & \\
\hline 205 & & & Thier aufgebunden. Kein Anaesthe- & \\
\hline $\begin{array}{l}206 \\
230\end{array}$ & & 38 & $\begin{array}{l}\text { Gell, etwas trübe } \\
\text { Operation zur Freilegung der Arteria }\end{array}$ & Kein Zucker \\
\hline 235 & & 3 & $\begin{array}{l}\text { axillaris dextra angefangen } \\
\text { Operation vollendet. Harn gelb, } \\
\text { trübe }\end{array}$ & Kein Zucker \\
\hline 242 & & & $\begin{array}{l}\text { Einspritzung der } 1 / 1 \text { molecu- } \\
\text { laren Harnstofflosung in } \\
\text { die Arteria axillaris an- } \\
\text { gefangen }\end{array}$ & \\
\hline 258 & & $21 / 2$ & Anfangende Haemoglobinurie & Kein Zucker \\
\hline 0.95 & 80 & $8^{1 / 2}$ & $\begin{array}{l}\text { Einspritzung aufgela oben } \\
\text { Schwere Haemoglobinurie }\end{array}$ & Kein Zucker \\
\hline 387 & & & $\begin{array}{l}\text { Einspritzungsfüssigkeit } \\
\text { gewechselt. Injection mit } \\
\text { 1/2 molecularer NaCl-Lö- } \\
\text { sungangefangen }\end{array}$ & \\
\hline $\begin{array}{ll}3 & 43 \\
3 & 45\end{array}$ & 27 & 8 & Tief roth, klar & Kein Zueker \\
\hline $\begin{array}{ll}3 & 47 \\
4 & 01 \\
4 & 06\end{array}$ & & ${ }^{7}$ & $\begin{array}{l}\text { Tief roth, klar } \\
\text { Thier ist cyanotisch. Harn tief roth }\end{array}$ & $\begin{array}{l}\text { Zucker } 0,25 \% \\
\text { Zucker } 0,31 \% \\
\text { Zucker } 0 \% 31 \%\end{array}$ \\
\hline 406 & & & Thier wird getödtet & Zucker $0,31 \%$ \\
\hline
\end{tabular}

\section{Zusammenfassung.}

1. Die Einspritzung von $75-100 \mathrm{ccm}$ pro 15 Minuten einer 1/6 molecularen NaCl-, NaBr-, NaI- oder $\mathrm{NaNO}_{3}$-Lösung in die Obrvenen oder in die oberflächlichen Venenzweige der Hüfte von Kaninchen ruft eine Polyurie und Glykosurie hervor. Die Polyurie kommt in 10-15 Minuten nach Beginn der Einspritzung zum Vorschein und dauert so lange, als die Finspritzung fortgesetzt wird (27 Stunden in einem Fall). Die Glykosurie tritt erst 2 Stunden nach Anfang der Salzeinflössung auf, und hält 6-8 Stunden an. Die Menge des im Harn ausgeschiedenen Zuckers ist zuerst klein, wächst aber schnell bis zu einem Maximum an, um allmählich wieder bis auf Null zu fallen. Der Procentsatz Zucker im Harn beim Maximum der 
Glykosurie liegt über $0,40 \%$. Nachdem die Glykosurie bis auf Null gefallen ist, bringt eine fortgesetzte Einflössung der Salzlösungen keine weitere Zuckerausscheidung herbei, obschon die Polyurie fortfährt.

2. Die Thatsache, dass der im Harn ausgeschiedene Procentsatz Zucker in diesen Versuchen nur selten über $0,40 \%$ steigt, veranschaulicht nicht die Schwere des Diabetes. Es muss vielmehr berủcksichtigt werden, dass in diesen Versuchen durch die Einflössung der grossen Mengen Wasser die Harnsecretion in der Zeiteinheit ungefähr 10-20 Mal so gross gemacht wird wie unter normalen Umständen. Dies besagt, dass wenn der Urin nicht in dieser Weise verdünnt wäre, die Zuckerausscheidung zu Zeiten $8 \%$ überschreitet.

3. Die intravenöse Einspritzung einer weniger als 1/6 molecularen Kochsalzlösung bringt zwar immer noch eime Polyurie hervor, aber eine Zuckerausscheidung kommt entweder gar nicht oder nur in Spuren und später zum Vorschein, als wenn dasselbe Quantum einer $1 / 6$ molecularen NaCl-Lösung eingeflösst wird. Wenn Kochsalzlösungen von einer höheren Concentration als $1 / 6$ moleculare eingespritzt werden, wird eine Glykosurie desto früher herbeigebracht, je höher die Coneentration der Salzlösung ist.

4. Die Zuckerausscheidung, die durch Einspritzung einer 1/6 molecularen NaCl-, NaBr-, NaI- oder $\mathrm{NaNO}_{3}$-Lösung hervorgerufen ist, kann herabgesetzt oder ganz gehemmt werden durch Zusatz von $\mathrm{CaCl}_{2}$ zu den reinen Natriumsalzlösungen $(975 \mathrm{ccm} 1 / 6$ moleculares Natriumsalz $+25 \mathrm{ccm} 3 / 8$ moleculares $\mathrm{CaCl}_{2}$ ).

5. Die Kaninchen unterscheiden sich in der Leichtigkeit, mit welcher eine Glykosurie in ihnen hervorgerufen oder gehemmt werden kann. Ich habe bis jetzt aber kein Kaninchen gefunden, in welchem nicht eine Glykosurie hervorgerufen werden konnte durch Einflössung 1/6 molecularer Lösungen von $\mathrm{NaCl}, \mathrm{NaBr}, \mathrm{NaI}$ oder $\mathrm{NaNO}_{3}$ (oder den noch weiter unten erwähnten Salzen), wenn dieselben nur in genügenden Mengen in der Zeiteinheit eingespritzt wurden.

6. Es existiert eine gewisse Latenzzeit, bevor die specifischen Wirkungen der verschiedenen Salze zum Vorschein kommen. Wenn z. B. eine Glykosurie durch eine reine $1 / 6$ moleculare NaCl-Lösung hervorgerufen ist, hört sie nicht früher auf als ein oder zwei Stunden nach Anfang der Einspritzung der $975 \mathrm{~cm}{ }^{1 / 6}$ molecularer NaCl- + $25 \mathrm{cem} 3 / 8$ molecularer $\mathrm{CaCl}_{2}$-Lösung. Nachdem die Zuckerausscheidung hierdurch aufgehoben ist, kann sie nicht wieder unter ein 
oder zwei Stunden von Neuem nach Rückkehr zu der reinen Kochsalzlösung hervorgerufen werden.

7. Eine Anzahl Experimente, in welchen der Versuch gemacht wurde, die Wirkung concentrirter Salzlösungen so weit wie möglich zu localisiren, seheinen darauf hinzuweisen, dass der Angriffspunkt all der wirksamen Salze in der Medulla oblongata liegt. Nach dieser Auffassung: ist die hier besprochene Glykosurie als ein Diabetes anzusehen, der durch Reizung des "Diabetes centrum" verursacht ist.

8. Wenn die Wirkung der Salzlösungen localisirt wird, genügen nur wenige Kubikcentimeter einer concentrirten Salzlösung um eine Glykosurie hervorzurufen. Diese Thatsache, und die verwendete Methode der Einspritzung erlaubten das Studium einer Anzahl Salze, die sich in früheren Versuchen als unbrauchbar erwiesen hatten, vermuthlich durch ihre giftige Wirkung auf das Herz. Ausser dem schon erwäbnten Natriumsalzen können auch die folgenden Salze eine Glyksurie in Kaninchen hervorrufen: $\mathrm{LiCl}, \mathrm{KCl}, \mathrm{SrCl}_{2}$. Als unwirksam erwiesen sich $\mathrm{NH}_{4} \mathrm{Cl}$ und als stets tödtlich $\mathrm{CaCl}_{2}$ und $\mathrm{MgCl}_{2}$.

9. Die Wirkung dieser Salze auf die Medulla ist nicht eine osmotische, sondern eine chemische oder pbysiko-chemische, denn die Einspritzung von Glycerin-, Harnstoff- oder AethylalkoholLösungen von derselben osmotischen Concentration, und in demselben und grösseren Beträgen als die Lösungen der wirksamen Salze bringt keine Glykosurie hervor. Sobald aber die Einspritzung dieser NichtElektrolyte durch die Einspritzung einer reinen $\mathrm{NaCl}$-Lösung ersetzt wird, kommt Zucker in der gewöhnlichen Weise im Harn zum Vorschein.

10. Der Procentsatz Zucker im Harn läuft der Menge des Urinwassers nicht etwa proportional eher umgekehrt. Indessen existirt kein festes Verhältniss zwischen der Menge des ausgeschiedenen Harns und der Menge des secernirten Zuckers, - beide variiren unabhängig voneinander. Es ist möglich eine schwere Glykosurie mit kaum einer Zunahme in der normalen Menge der Harnsecretion zu bekommen. Diese Thatsachen sprechen gegen die Idee gewisser Forscher, die die Ursache der Zuckerausscheidung nach Einspritzung verschiedener Diuretica (einschliesslich reiner Kochsalzlösungen) in der gesteigerten Harnsecretion suchen.

11. Die Glykosurie ist öfters aber durchaus nicht immer von einer Albuminurie begleitet. 